\title{
COVIDSAVIOR: A Novel Sensor-Fusion and Deep Learning Based Framework for Virus Outbreaks
}

\section{OPEN ACCESS}

Edited by:

Thippa Reddy Gadekallu,

VIT University, India

Reviewed by:

Pooja Shah,

Gandhinagar Institute of Technology,

India

Abdul Rehman Javed,

Air University, Pakistan

Chennareddy Vijay Simha Reddy,

Middlesex University, United Kingdom

*Correspondence:

Sharnil Pandya

sharnil.pandya@sitpune.edu.in

Specialty section:

This article was submitted to

Digital Public Health,

a section of the journal

Frontiers in Public Health

Received: 19 October 2021 Accepted: 02 November 2021 Published: 30 November 2021

Citation:

Pandya S, Sur A and Solke N (2021) COVIDSAVIOR: A Novel Sensor-Fusion and Deep Learning Based Framework for Virus Outbreaks.

Front. Public Health 9:797808. doi: 10.3389/fpubh.2021.797808

\author{
Sharnil Pandya*, Anirban Sur and Nitin Solke \\ Symbiosis Institute of Technology, Symbiosis International (Deemed) University, Pune, India
}

The presented deep learning and sensor-fusion based assistive technology (Smart Facemask and Thermal scanning kiosk) will protect the individual using auto face-mask detection and auto thermal scanning to detect the current body temperature. Furthermore, the presented system also facilitates a variety of notifications, such as an alarm, if an individual is not wearing a mask and detects thermal temperature beyond the standard body temperature threshold, such as $98.6^{\circ} \mathrm{F}\left(37^{\circ} \mathrm{C}\right)$. Design/methodology/approach-The presented deep Learning and sensor-fusion-based approach can also detect an individual in with or without mask situations and provide appropriate notification to the security personnel by raising the alarm. Moreover, the smart tunnel is also equipped with a thermal sensing unit embedded with a camera, which can detect the real-time body temperature of an individual concerning the prescribed body temperature limits as prescribed by WHO reports. Findings - The investigation results validate the performance evaluation of the presented smart face-mask and thermal scanning mechanism. The presented system can also detect an outsider entering the building with or without mask condition and be aware of the security control room by raising appropriate alarms. Furthermore, the presented smart epidemic tunnel is embedded with an intelligent algorithm that can perform real-time thermal scanning of an individual and store essential information in a cloud platform, such as Google firebase. Thus, the proposed system favors society by saving time and helps in lowering the spread of coronavirus.

\footnotetext{
Keywords: deep learning, sensor-fusion, COVID-19, novel corona virus, auto-face mask detection, auto-thermal scanning, YOLOv4, body temperature
}

\section{INTRODUCTION}

The COVID-19 outbreak has given sleepless nights to the entire world for the last 6 months. In recent times, an economy-driven country such as India has recorded more than 1,500,000 cases (1). Considering these facts, researchers are putting enormous efforts into developing innovative solutions to deal with the current pandemic. Furthermore, according to WHO reports, the 
WHO has put enormous efforts into assisting various developed and developing countries in terms of masks, ventilators, hospital beds, face shields, and other essential health equipments (2). Furthermore, the WHO has also prepared strict guidelines for lock-downs, social-distancing, and testing of COVID-19 suspects. In developing countries, such as India, along with the physical efforts of doctors, nurses, and the paramedical staff, the government has utilized digital technologies in a variety of ways, such as "Aarogya-Setu app," which can assist citizens in identifying near-by COVID-19 patients using Bluetooth and GPS based remote tracking technologies. However, looking at the impact and the spread of coronavirus, fellow researchers need to put more effort into designing and developing COVID19 solutions (3-7). It is a fact that fellow researchers have proposed various innovative approaches to deal with the current precarious situation, including automatic sanitizer systems for the disinfection of medical equipment and individuals, thermal scanning guns, and many more. However, the development of COVID-19 assistive systems has remained an open issue for fellow researchers (8-10). In addition to this, various state governments have formulated strict health prevention policies and have made genuine efforts to disinfect various geographical regions using various sanitizers, such as disinfectants and insecticides. The government has also executed numerous health-awareness campaigns for the well-being of society. In some cases, the government has also penalized mischievous individuals who have attempted to break the health and safety procedures. It has also been recorded that the police have to patrol in the surrounding areas to ensure strict adherence to health and safety precautions, such as wearing a mask, maintaining social distancing etc. However, it is also observed and predicted by the world's health experts that society will not have access to coronavirus vaccination in the near future. So the fellow researchers need to keep on developing efficient and effective solutions to avoid unwanted circumstances (11). For this purpose, we have carried out a detailed and rigorous analysis of the existing methodologies and identified certain research gaps. It is also imperative that if the situation continues to persist for a longer time, we have to learn to live with the given problems by keeping ourselves and sound at the workplace. To resolve the discussed issues, in the undertaken study, we have presented a deep learning and sensor-fusionbased approach for detecting a face mask and real-time body temperature for COVID-19, which is term as "Smart Facemask and Thermal scanning kiosk" throughout this paper. The presented deep learning and sensor-fusion-based approach is designed to detect an individual with or without mask conditions and notify security personnel by raising the alarm. Moreover, the smart tunnel is also equipped with a thermal sensing unit embedded with a camera, which can detect the real-time body temperature of an individual concerning the prescribed body temperature limits as prescribed by WHO reports. The presented article is organized as follows: Section 2 discusses the state of the art methodologies. Section 3 discusses the necessity of the presented system, section 4 and 5 discusses the design and experimental setup, architecture design, sensing arrangements, deployments, and the detailed workflow of the presented system. Finally, section 6 provides the concluding remarks.

\section{RELATED WORK}

Yang and his team have proposed an object detection automasking neural network for capturing discriminative objects. In this study, a variety of simulation-based study has been carried out. The proposed system is not tested in a realtime environment (12). Joshi R. has presented a health kiosk model using Computational Fluid Dynamics (CFD) simulationbased studies. However, the presented approach has been tested under the simulation environment only (13). Fan and his fellow researchers have proposed an auto-lung segmentation system to detect CT-scans of coronavirus patients. However, the proposed system did not facilitate the scanning of face-masks and the body temperature of humans (14). Maurya and his team have presented an innovative method for disinfecting humans. However, the proposed system did not facilitate any kind of facemask and body temperature scanning (15). Ghayvat and his team have presented a system to keep the track of social-distancing policies between two persons. For this purpose, the proposed system has used smart cities, such as ITS infrastructures. However, the proposed system did not discuss any kind of scanning approach for the human body and face-masks (2). Abbas and his fellow mates have presented a chest X-ray scanning system of COVID-19 suspects. However, the presented system is not capable to do thermal scanning of a human body or identifying obscured faces (16). Apostolopoulos and Mpesiana have presented a chest X-ray scanning system of COVID-19 suspects. However, the presented systems were not capable to do thermal scanning of a human body or identifying obscured faces (17). Poon and his research team have proposed a system that is capable to carry out obstetric and gynecological scans. The main purpose of the presented system was to do scanning of medical equipment (18). Kim and Lee have presented a respiratory illness scanning system to detect diseases, such as COPD. However, the proposed system did not facilitate thermal scanning and the detection of obscured faces (19). Ucar and fellow researchers have presented an AI-based CT-scan scanning system to detect coronavirus symptoms. However, the presented system did not discuss anything like detecting body temperature or face-masks (20). Kwon and fellow researchers have presented a human scanning system to detect COVID-19 like symptoms for a drive-through. However, the presented system did not discuss anything like detecting body temperature or face-masks (21). Majid et al. (22) researchers have presented a sensor-fusion based wearable device that can scan and disinfect human hands from coronavirus infections (22). Takagi and Yagishita have presented a detailed comparison and discussion of various health and safety policies related to COVID-19 issues. However, the system did not propose any system for COVID-19 like diseases (23). Farman and team have presented a geofencing based realtime tracking system for COVID-19 patients. However, the presented system did not discuss any scanning mechanisms for the current pandemic situations. However, the system was not 
designed for the disinfection of healthy patients (24). Lippi and fellow researchers have analyzed and discussed numerous biosafety policies and precautions. However, the presented system did not discuss any scanning approaches for humans (25). Mahammedi et al. (26) have presented a novel solar powerbased system to disinfect things such as mobile, key, wallet, and many more. However, the system did not possess the capability to perform any kind of scanning. Pandya et al. (27) have presented a novel face-detection system that can detect a human with fully or partially covered faces. However, the system did not possess the capability to detect face-masks. Ghayvat et al. (28) have presented a sensor-fusion based smart aging system to monitor the elderly well beings. However, the system did not facilitate the scanning of faces and body temperature of a human.

\section{NECESSITY OF A SMART FACE MASK AND THERMAL SCANNING KIOSK}

Recent fellow researchers have made efforts to implement a costeffective pandemic tunnel to disinfect humans from COVID-19 like infections. In this study, an IoT based sensor fusion assistive tunnel has been presented, which can disinfect an individual from the possibility of coronavirus infections. The major contributions of the presented system are as follows: a deep learning and IoT based sensor fusion assistive framework has been proposed to do real-time detection of individuals with face-mask and without face-mask conditions. In the presented research work, a solarpowered smart tunnel has been presented, installed and deployed at the entrance of the Symbiosis institute of technology, Pune. The contributions of the proposed work are as follows:
1. The presented system can also detect an outsider who is entering the building with or without mask conditions using You Only Look Once 4 (YOLOv4) computer vision algorithm (29) and notifies the security control room by raising appropriate alarms.

2. The presented smart face-mask detection and thermal scanning kiosk can function using solar energy during the day, and it functions using a solar power bank at night time. This functionality has been provided by light-dependent resistor (LDR) sensing unit placed in a tunnel.

3. The smart tunnel also provides the facility to do real-time attendance of all the staff entering the smart tunnel, along with thermal scanning and face-mask detection.

4. In the end, web and mobile interface has been designed to provide daily, weekly and monthly reports of the recorded body temperatures of individuals, along with inout timestamp values. The investigation results validate the performance evaluation of the presented smart face-mask and thermal scanning kiosk. The presented smart tunnel is embedded with an intelligent algorithm that can perform realtime thermal scanning of an individual and stores the essential information in a cloud platform, such as google firebase.

\section{DESIGN AND EXPERIMENTAL SETUP}

In the experimental setup, each sensor is placed in different positions in the house, with a single ESP8266 attached to it, where the basic data processing takes place. ESP8266 comes with a Wi-Fi module attached to it, using which the processed data is then transferred logged into the Raspberry Pi B+ server. To avoid data duplication of erroneous data, pre-processing of sensor data is done at the ESP8266 level, thus ensuring
A

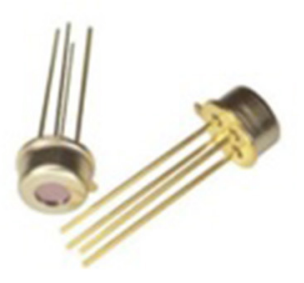

D

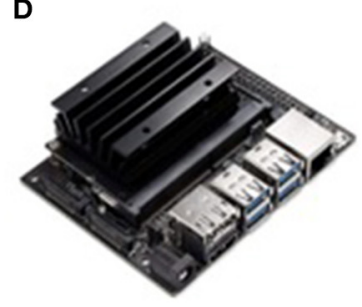

B

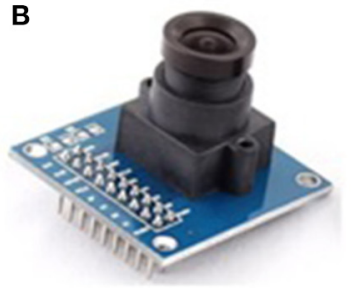

E

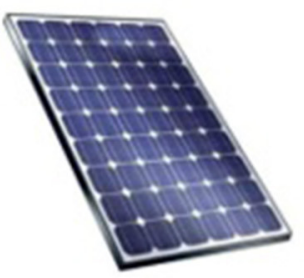

C

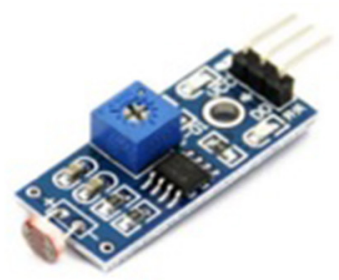

$\mathbf{F}$

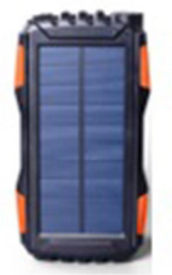

FIGURE 1 | Design and experimental setup of a smart epidemic tunnel (A) thermopile temperature sensor a Robocraze OV7670 (B) 300 KP VGA Camera (C) an LDR sensing unit (D) a NVIDIA Jetson Nano (E) a solar cell (F) a solar power bank. 
A

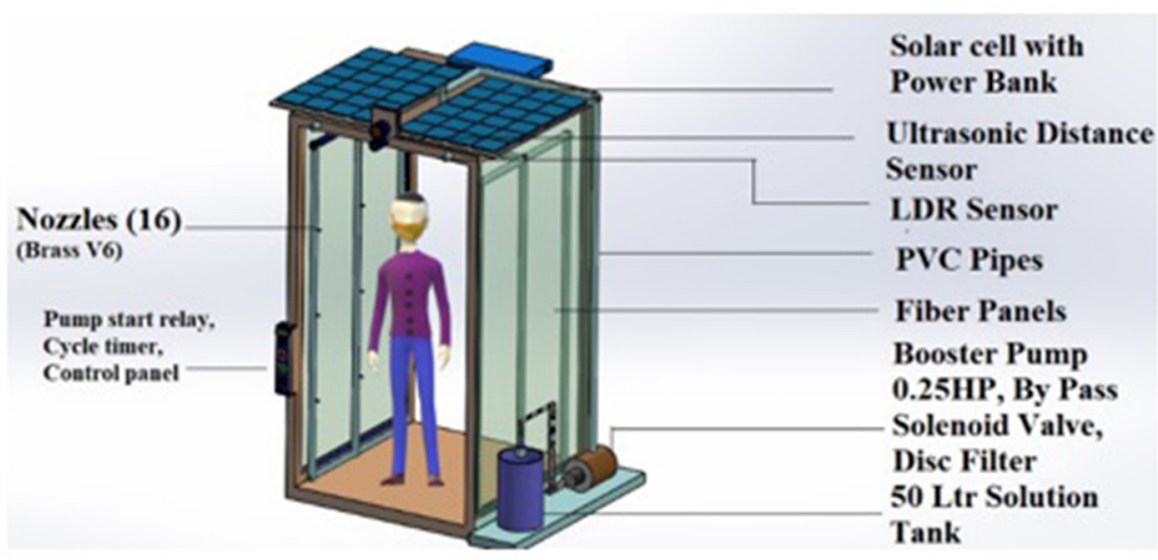

B

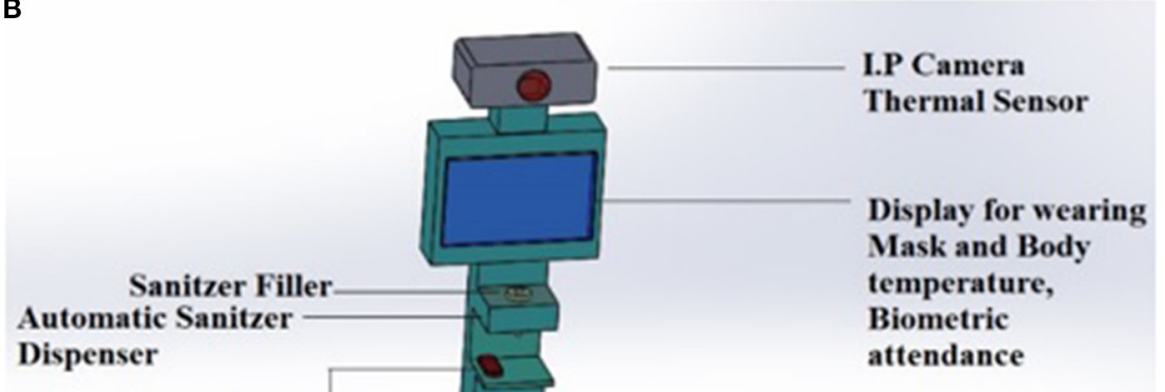

Finger Scanner and Temperature Sensor

Adjustable Stand

FIGURE 2 | Prototype design of a (A) smart epidemic tunnel (B) face-mask and thermal scanning Kiosk.

that Raspberry pi is only used as the local server where clean data is stored in the whole setup. Logged Data is periodically sent to the Cloud storage to ensure backup of data is kept in case of any system failure, along with the logs of whole systems working, which can also be later on utilized for systems debugging. The presented smart tunnel is an extended version of the smart epidemic tunnel, which had facilitated the ultrasonic sensor-based sanitizer system and timestampbased notifications. In the presented research work, we have proposed a customized smart face-mask and thermal scanning kiosk equipped in a previously presented smart epidemic tunnel. The presented system is flexible enough to be placed outside public spots such as malls, university buildings, companies, busstop, hospital ICU units, vegetable markets, railway stations, and many more. Figures 1A-F represent a design and experimental setup used in the conducted experiments such as a Thermopylae sensor, a VGA camera, LDR sensing unit, Netson NVIDIA controller, a solar cell, and a solar power bank. Figure 2 represents a prototype design of the presented smart face mask and thermal scanning kiosk equipped in a smart epidemic tunnel.

\section{METHODOLOGY}

Fellow researchers have made efforts to implement a costeffective pandemic tunnel to disinfect humans from COVID19 like infections. In this study, an IoT based sensor fusion assistive tunnel has been presented, which can disinfect an individual from the possibility of coronavirus infections. The major contributions of the presented PWP system are as follows: (i) a deep learning and IoT based sensor fusion assistive framework have been proposed to do real-time detection of individuals with face-mask and without face-mask conditions. In the presented research work, a solar-powered smart tunnel has been presented, installed, and deployed at the entrance of the Symbiosis institute of technology, Pune. (i) The presented system can also detect an outsider who is entering the building with or without mask conditions using YOLOv4 computer vision algorithm and notifies the security control room by raising appropriate alarms. (ii) The presented smart face-mask detection and thermal scanning kiosk can function using solar energy during the day, and it functions using a solar power bank at night. This functionality has been provided by an (LDR) sensing unit 


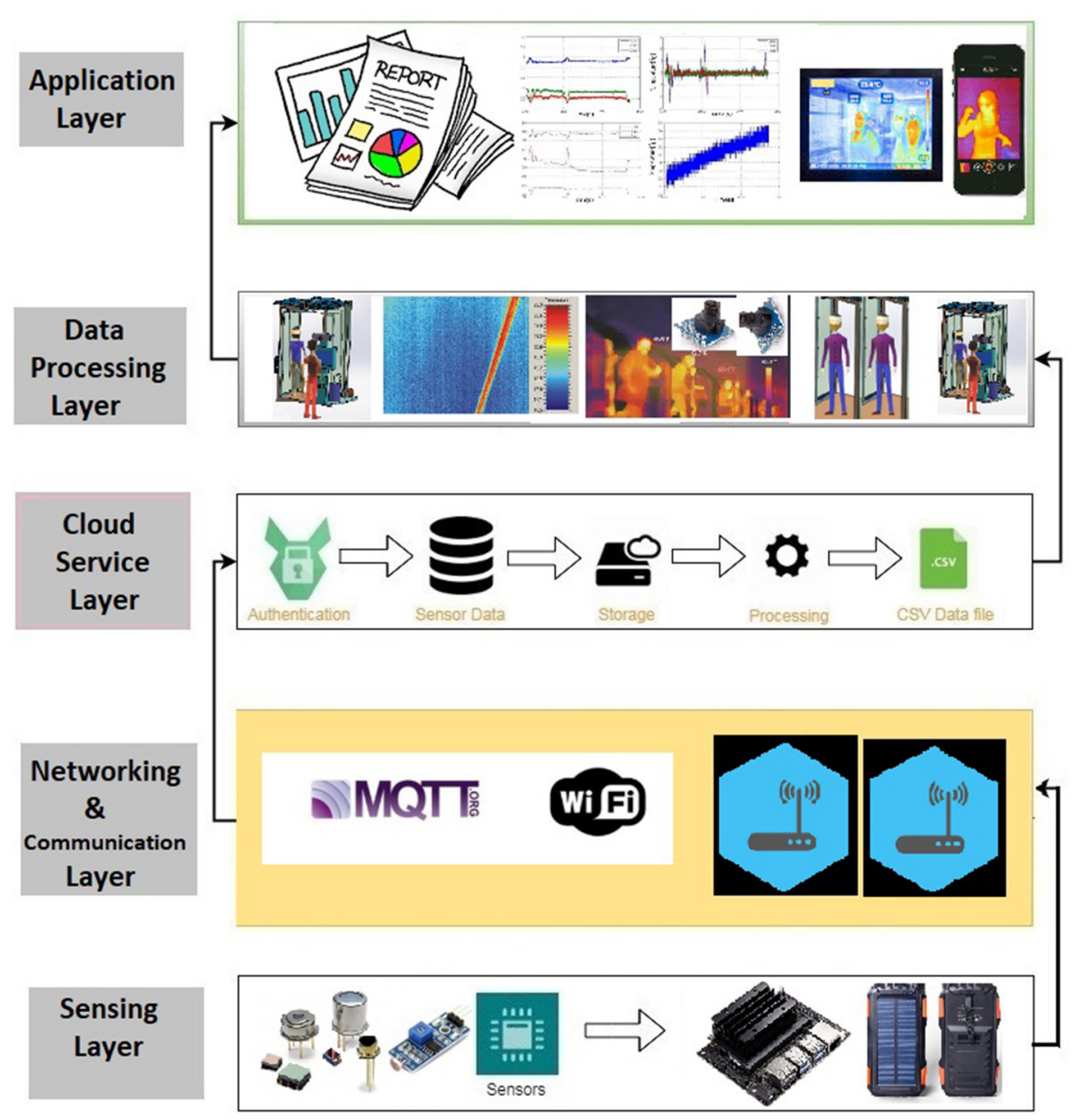

FIGURE 3 | An architectural design of a smart facemask and thermal scanning system prototype design of a facemask and thermal scanning Kiosk.

placed in a tunnel. (iii) The smart tunnel also provides the facility to do real-time attendance of all the staff entering the smart tunnel, along with thermal scanning and face-mask detection. (iv) In the end, web and mobile interface has been designed to provide daily, weekly, and monthly reports of the recorded body temperatures of individuals, along with in-out timestamp values. The investigation results validate the performance evaluation of the presented smart face-mask and thermal scanning kiosk. The presented smart tunnel is embedded with an intelligent algorithm that can perform real-time thermal scanning of an individual and stored the essential information in a cloud platform, such as google firebase. In the pandemic situation, a smart face-mask and thermal scanning kiosk have been presented, equipped in a smart epidemic tunnel. The presented research work is an extended version of the smart epidemic tunnel. In the presented research work, we have added certain novel features to enhance the presented system's performance. The presented deep learning and sensor-fusion based assistive technology (Smart Facemask and Thermal scanning kiosk) will protect the individual using auto face-mask detection and auto thermal scanning to detect the current body temperature. The presented smart tunnel is embedded with an intelligent algorithm that can perform real-time thermal scanning of an individual and stored the essential information in a cloud platform, such as google firebase. Furthermore, in the conducted experiments, the YOLOv4 computer vision algorithm has been applied to detect individuals with face-mask and without faced-mask conditions. Furthermore, the presented system also facilitates a variety of notifications such as an alarm if an individual is not wearing a mask and detects thermal temperature beyond the standard body temperature threshold such as $98.6^{\circ} \mathrm{F}\left(37^{\circ} \mathrm{C}\right)$. Furthermore, a solar power bank is also used for storing solar energy, which the proposed system will utilize at night. In the end, the investigation results validate the performance evaluation of the presented smart face-mask and thermal scanning kiosk.

\subsection{The Layered Design of a Smart Face-Mask and Thermal Scanning Kiosk}

Figure 3 represents an architectural design of the presented novel deep Learning and sensor-fusion-based approach for detecting 

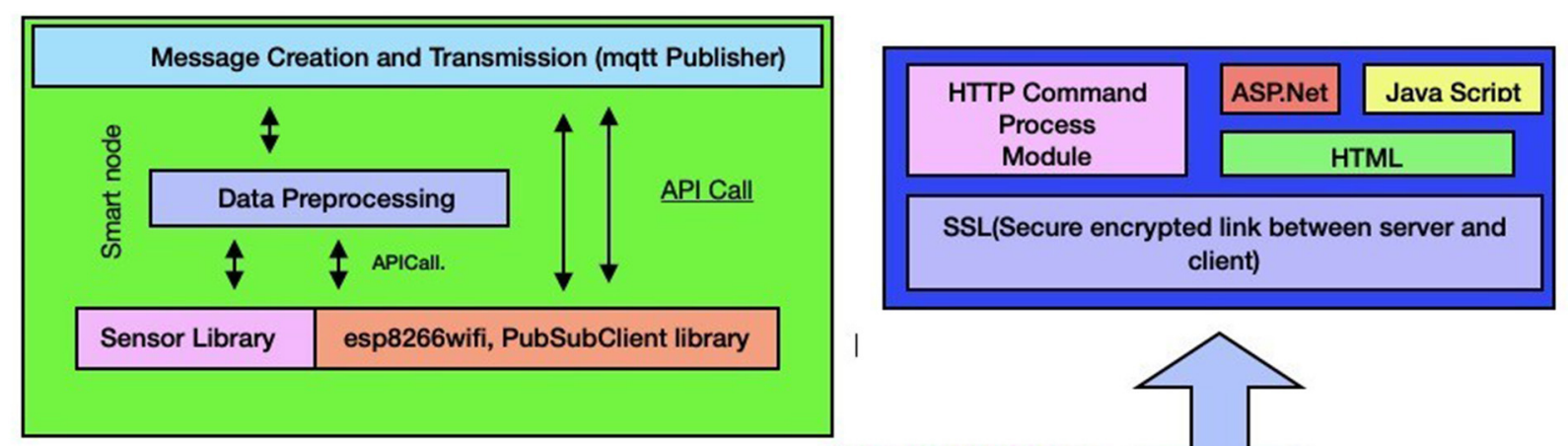

।
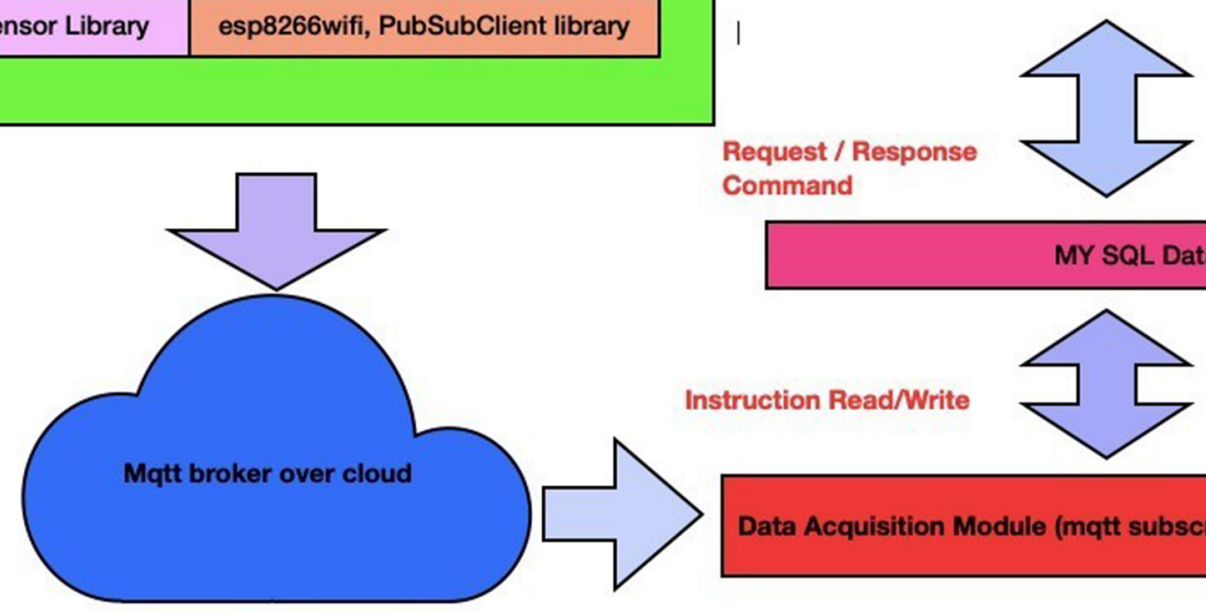

Request / Response

Command

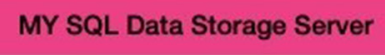

Instruction Read/Write

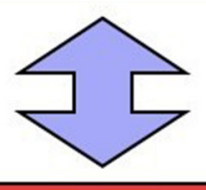

Data Acquisition Module (mqtt subscriber : Paho-mqtt Client)

FIGURE 4 | A back-end design of a smart face-mask thermal scanning system.

a face mask and real-time body temperature for COVID-19 outbreak situations. The extended version of the presented has been placed at the Symbiosis Institute of Technology, Pune, which has been implemented for performing auto-face mask detection and thermal scanning of various Symbiosis stakeholders such as faculty members, students, and external visitors. The presented architectural design contains five layers: (i) physical (sensing) layer, (ii) communication and networking layer, (iii) cloud computing (google firebase storage) layer, (iv) data processing layer, and (v) application layer. The main purpose of the physical sensing layer is to detect the body temperature of individuals who are present in the projection areas of a VGA camera equipped in the presented thermal scanning kiosk. This sensing layer contains various sensing units such as the LDR sensing unit and a thermopile temperature sensor. Furthermore, this layer also contains an intelligent NVIDIA Netson controller, along with a solar cell and a solar power bank. A YOLOv4 computer vision algorithm has been installed in an NVIDIA Netson controller to detect humans with face masks and without face-mask conditions. In addition to this, this layer also facilitates a solar power backup using a solar cell during the day-time and a solar power bank during the night time. Again, in the day-time, the presented smart face-mask and thermal scanning kiosk start functioning using a solar cell.

\subsubsection{Sensing Layer}

The main purpose of the physical sensing layer is to detect the body temperature of individuals present in the projection areas of a VGA camera equipped in the presented thermal scanning kiosk. This sensing layer contains various sensing units, such as the LDR sensing unit and a thermopile temperature sensor. Furthermore, this layer also contains an intelligent NVIDIA Netson controller, along with a solar cell and a solar power bank. A YOLOv4 computer vision algorithm has been installed in an NVIDIA Netson controller to detect humans with face masks and without face-mask conditions. In addition to this, this layer also facilitates a solar power backup using a solar cell during daytime and a solar power bank during nighttime. Again, in the daytime, the presented smart face-mask and thermal scanning kiosk start functioning using a solar cell.

\subsubsection{Communication and Networking Layer}

The networking and communication layer is responsible for interfacing and transmitting information between a physical(sensing) layer, cloud services layer, and an application layer using a broker architecture of an MQTT protocol. Furthermore, it transmits scanned body temperature values, timestamps, and the count of people wearing or not wearing a face mask on a google firebase platform. Figure 4 represents a back-end design of a smart epidemic tunnel.

\subsubsection{Communication and Networking Layer}

The networking and communication layer is responsible for interfacing and transmitting information between a physical(sensing) layer, cloud services layer, and an application layer using a broker architecture of an MQTT protocol. 
Furthermore, it transmits scanned body temperature values, timestamps, and the count of people wearing or not wearing a face mask on a google firebase platform. Figure 4 represents a back-end design of a smart epidemic tunnel.

\subsubsection{Cloud Computing (Google Firebase) Layer}

The cloud computing layer facilitates the storage of the temperature values of individuals scanned using a thermal sensing unit with timestamp values and the count of people with face-mask and without face-mask variations. Furthermore, it keeps track of various individuals who have accessed the smart face-mask and thermal scanning kiosk during the day or night timings. In the conducted experiments, the Google Firebase cloud computing platform has been used for storage purposes.

\subsubsection{Processing Layer}

The processing layer fetches the temperature and face-mask related values from the Google Firebase database via MQTT broker architecture. This layer performs two essential operations: (i) the processing layer detects the body temperature of individuals using a thermal sensor placed in a smart thermal scanning kiosk (ii) it also facilitates two essential bifurcation of the people who are wearing or not wearing face-masks using a YOLOv4 computer vision algorithm deployed in an NVIDIA Netson controller. Eventually, the processed information will be passed on to the application layer for generating various reports.

\subsubsection{Application Layer}

The application layer received organized information from the processing layer and represented it in various graphical and tabular representations. Furthermore, the application layer also consists of the web and mobile interface, which provides daily, weekly, and monthly updates such as timestamp-based temperature detection reports, the count of people wearing or not wearing face-masks, and the number of individuals who have access to the presented face-mask and thermal scanning kiosk during the day and night timings.

\section{THE DETAIL WORKING OF THE FACE-MASK AND THERMAL SCANNING KIOSK}

As shown in Figure 5, the presented smart system performs two key operations during this phase: (i) it also facilitates the bifurcation of the people based on a variety of face mask conditions such as with and without face mask conditions using a YOLOv4 computer vision algorithm deployed in an NVIDIA Netson controller. (ii) The presented smart system can detect the body temperature of individuals present in the projection areas of a Robocraze OV7670 300KP VGA camera using a thermopile temperature sensing unit.

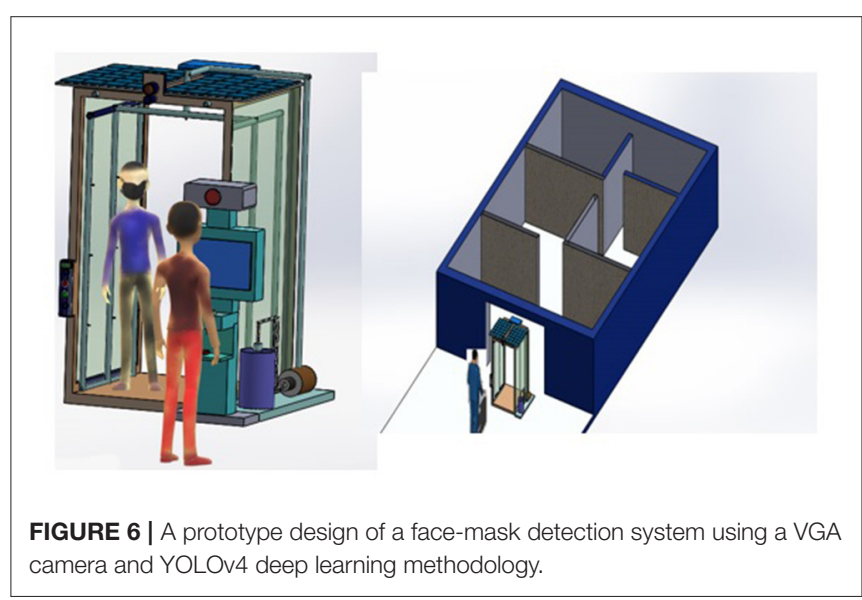

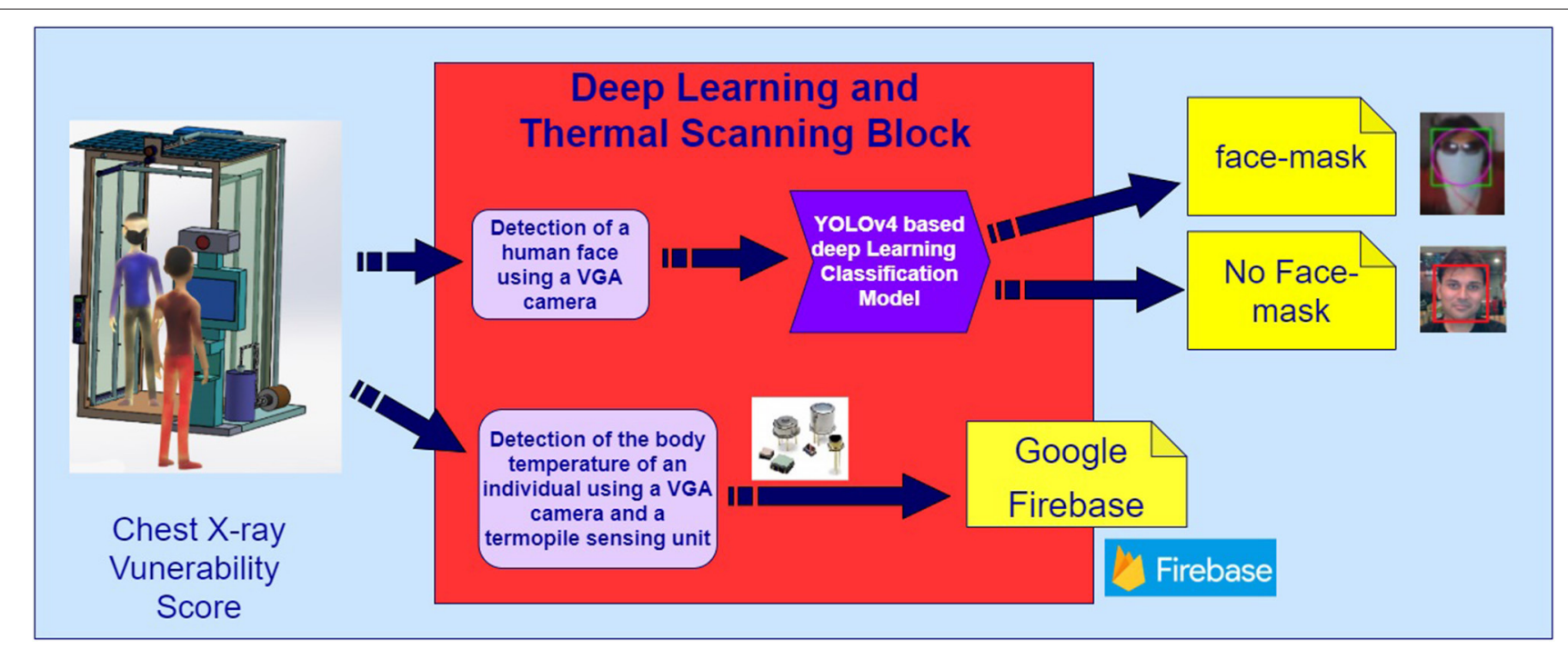

FIGURE 5 | A topology design of a smart facemask and thermal scanning system. 


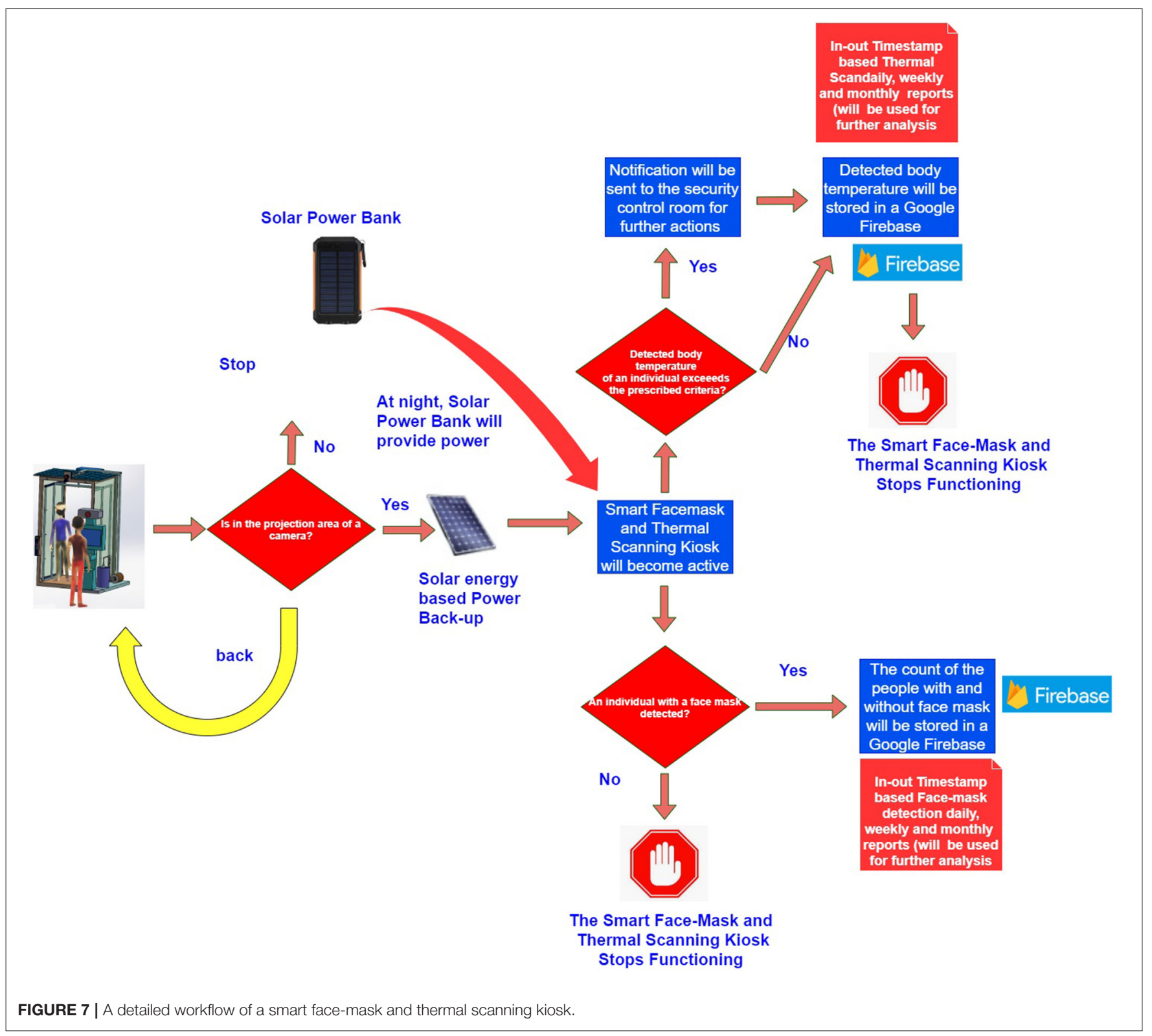

\subsection{The Detailed Working of the Face-Mask Detection Methodology}

The face-mask detection feature becomes active when an individual comes into the projection area of a VGA camera, as shown in Figure 6. In the conducted experiments, we have used a customized dataset of 1,000 face samples. After an individual is detected by a VGA camera placed in a smart kiosk, a YOLOv4 computer vision algorithm deployed in an NVIDIA Netson controller process the captured frame; during this phase, a YOLOv4 algorithm makes the use of cross-stage partial connection and separates the feature maps in two different parts: (i) the first part of the feature map bypasses the dense block and directly moves toward the transition block. (ii) The second part went through the dense block and reached the transition block. This kind of design increases the efficiency of the YOLOv4 algorithm by reducing the complexity of the presented approach. In addition to this, the YOLOV4 algorithm also utilizes crossstage-partial connections with CSPDarknet-53 feature extraction methodology, which enables the presented algorithm to achieve very high objection detection accuracy. Figure 7 represents a YOLOv4 methodology, and Figure 8 represents a detailed workflow of the presented smart face-detection and thermal scanning kiosk. As shown in Figure 8, after an individual is detected by a VGA camera placed in a smart kiosk using a YOLOv4 computer vision algorithm, it will process the captured frame of an individual to identify whether the captured individual is wearing a face-mask to protect against COVID-19 or not. Then, the algorithm will transmit the processed information to 


\begin{tabular}{|c|c|c|c|c|c|c|c|c|c|c|c|c|c|c|c|c|c|c|c|}
\hline yer & & Filters & & & input & & & output & & & & & & & & & & & \\
\hline 0 & conv & 32 & $3 \times 3 / 1$ & $608 \times$ & $608 \times 3$ & $\Rightarrow$ & $608 \times$ & $608 \times 32$ & 0.639 BFLOPs & 58 & res & 55 & & $38 \times$ & $38 \times 512$ & $\rightarrow$ & $38 \times$ & $38 \times 512$ & \\
\hline & conv & 64 & $3 \times 3 / 2$ & $608 \times$ & $608 \times 32$ & $->$ & $304 x$ & $304 \times 64$ & 3.407 BFLOPS & & & 256 & $1 \times 1 / 1$ & $38 \times$ & $38 \times 512$ & $\Rightarrow$ & $38 \times$ & $38 \times 256$ & 0.379 BFLOPS \\
\hline & conv & 32 & $1 \times 1 / 1$ & $304 \times$ & $304 \times 64$ & $\rightarrow$ & $304 \times$ & $304 \times 32$ & 0.379 BFLOPS & & conv & 512 & $3 \times 3 / 1$ & $38 \times$ & $38 \times 256$ & $\Rightarrow$ & $38 \times$ & $38 \times 512$ & 3.407 BFLOPS \\
\hline & & 64 & $3 \times 3 / 1$ & $304 \times$ & $304 \times 32$ & $\rightarrow$ & $304 x$ & $304 \times 64$ & 3.407 BFLOPS & 61 & res & 58 & & $38 \times$ & $38 \times 512$ & $\rightarrow$ & $38 \times$ & $38 \times 512$ & \\
\hline 4 & & 1 & & $304 \times$ & $304 \times 64$ & $\rightarrow$ & $304 x$ & $304 \times 64$ & & & conv & 1024 & $3 \times 3 / 2$ & $38 \times$ & $38 \times 512$ & $->$ & $19 \times$ & $19 \times 1024$ & 3.407 BFLOPS \\
\hline & & 128 & $3 \times 3 / 2$ & $304 \times$ & $304 \times 64$ & $\rightarrow$ & $152 \times$ & $152 \times 128$ & 3.407 BFLOPs & 63 & & 512 & $1 \times 1 / 1$ & $19 \times$ & $19 \times 1024$ & $\rightarrow$ & $19 \times$ & $19 \times 512$ & 0.379 BFLOPS \\
\hline & & 64 & $1 \times 1 / 1$ & $152 \times$ & $152 \times 128$ & $\rightarrow>$ & $152 \times$ & $152 \times 64$ & 0.379 BFLOPS & & conv & 1024 & $3 \times 3 / 1$ & $19 \times$ & $19 \times 512$ & $\rightarrow$ & $19 \times$ & $19 \times 1024$ & $3.407 \mathrm{BFLOPS}$ \\
\hline & & 128 & $3 \times 3 / 1$ & & $152 \times 64$ & $\rightarrow$ & $152 \times$ & $152 \times 128$ & 3.407 BFLOPS & 65 & res & 62 & & $19 \times$ & $19 \times 1024$ & $\rightarrow$ & $19 \times$ & $19 \times 1024$ & \\
\hline & & 5 & & $152 \times$ & $152 \times 128$ & $\Rightarrow$ & $152 \times$ & $152 \times 128$ & & 66 & conv & 512 & $1 \times 1 / 1$ & $19 \times$ & $19 \times 1024$ & $\rightarrow$ & $19 \times$ & $19 \times 512$ & 0.379 BFLOPS \\
\hline & & 64 & $1 \times 1 / 1$ & $152 \times$ & $152 \times 128$ & $->$ & $152 \times$ & $152 \times 64$ & 0.379 BFLOPs & & conv & 1024 & $3 \times 3 / 1$ & $19 \times$ & $19 \times 512$ & $\rightarrow$ & $19 x$ & $19 \times 1024$ & 3.407 BFLOPS \\
\hline 10 & & 128 & $3 \times 3 / 1$ & $152 \times$ & $152 \times 64$ & $\Rightarrow$ & $152 \times$ & $152 \times 128$ & 3.407 BFLOPS & 68 & res & 65 & & $19 \times$ & $19 \times 1024$ & $\rightarrow$ & $19 \times$ & $19 \times 1024$ & \\
\hline 11 & & 8 & & $152 \times$ & $152 \times 128$ & $\Rightarrow$ & $152 \times$ & $152 \times 128$ & & 69 & conv & 512 & $1 \times 1 / 1$ & $19 \times$ & $19 \times 1024$ & $\rightarrow$ & $19 x$ & $19 \times 512$ & 0.379 BFLOPS \\
\hline 12 & & 256 & $3 \times 3 / 2$ & $152 \times$ & $152 \times 128$ & $->$ & $76 \times$ & $76 \times 256$ & 3.407 BFLOPs & 70 & conv & 1024 & $3 \times 3 / 1$ & $19 \times$ & $19 \times 512$ & $->$ & $19 \times$ & $19 \times 1024$ & 3.407 BFLOPS \\
\hline 13 & & 128 & $1 \times 1 / 1$ & $76 \times$ & $76 \times 256$ & $\Rightarrow$ & $76 \times$ & $76 \times 128$ & 0.379 BFLOPS & 71 & res & 68 & & $19 \times$ & $19 \times 1024$ & $\rightarrow$ & $19 \times$ & $19 \times 1024$ & \\
\hline 14 & & 256 & $3 \times 3 / 1$ & $76 \times$ & $76 \times 128$ & $\Rightarrow$ & $76 x$ & $76 \times 256$ & 3.407 BFLOPs & 72 & conv & 512 & $1 \times 1 / 1$ & $19 \times$ & $19 \times 1024$ & $\rightarrow$ & $19 x$ & $19 \times 512$ & $0.379 \mathrm{BFLOPS}$ \\
\hline 15 & & 12 & & $76 \times$ & $76 \times 256$ & $\Rightarrow$ & $76 \times$ & $76 \times 256$ & & 73 & conv & 1024 & $3 \times 3 / 1$ & $19 \times$ & $19 \times 512$ & $\rightarrow$ & $19 x$ & $19 \times 1024$ & 3.407 BFLOPS \\
\hline 16 & & 128 & $1 \times 1 / 1$ & $76 \times$ & $76 \times 256$ & $\Rightarrow$ & $76 \times$ & $76 \times 128$ & 0.379 BFLOPS & 74 & res & 71 & & $19 \times$ & $19 \times 1024$ & $\rightarrow$ & $19 \mathrm{x}$ & $19 \times 1024$ & \\
\hline 17 & conv & 256 & $3 \times 3 / 1$ & $76 x$ & $76 \times 128$ & $->$ & $76 x$ & $76 \times 256$ & 3.407 BFLOPs & 75 & conv & 512 & $1 \times 1 / 1$ & $19 \times$ & $19 \times 1024$ & $\rightarrow$ & $19 x$ & $19 \times 512$ & 0.379 BFLOPS \\
\hline 18 & & 15 & 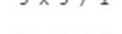 & $76 \times$ & $76 \times 256$ & $\rightarrow$ & $76 \times$ & $76 \times 256$ & & 76 & conv & 1024 & $3 \times 3 / 1$ & $19 \times$ & $19 \times 512$ & $\rightarrow$ & $19 x$ & $19 \times 1024$ & 3.407 BFLOPS \\
\hline 19 & conv & 128 & $1 \times 1 / 1$ & $76 \times$ & $76 \times 256$ & $\Rightarrow$ & $76 \times$ & $76 \times 128$ & 0.379 BFLOPs & 77 & conv & 512 & $1 \times 1 / 1$ & $19 \times$ & $19 \times 1024$ & $\rightarrow$ & $19 x$ & $19 \times 512$ & 0.379 BFLOPS \\
\hline 20 & conv & 256 & $3 \times 3 / 1$ & $76 x$ & $76 \times 128$ & $->$ & $76 x$ & $76 \times 256$ & 3.407 BFLOPs & 78 & conv & 1024 & $3 \times 3 / 1$ & $19 \times$ & $19 \times 512$ & $->$ & $19 x$ & $19 \times 1024$ & 3.407 BFLOPS \\
\hline 21 & & 18 & & $76 \times$ & $76 \times 256$ & $\Rightarrow$ & $76 \times$ & $76 \times 256$ & & 79 & conv & 512 & $1 \times 1 / 1$ & $19 \times$ & $19 \times 1024$ & $\rightarrow$ & $19 \times$ & $19 \times 512$ & 0.379 BFLOPS \\
\hline 22 & conv & 128 & $1 \times 1 / 1$ & $76 x$ & $76 \times 256$ & $->$ & $76 x$ & $76 \times 128$ & 0.379 BFLOPS & 80 & conv & 1024 & $3 \times 3 / 1$ & $19 \times$ & $19 \times 512$ & $\Rightarrow$ & $19 \mathrm{x}$ & $19 \times 1024$ & 3.407 BFLOPS \\
\hline 23 & conv & 256 & $3 \times 3 / 1$ & $76 \times$ & $76 \times 128$ & $\stackrel{->}{ }$ & $76 \times$ & $76 \times 256$ & 3.407 BFLOPs & 81 & conv & 255 & $1 \times 1 / 1$ & $19 \times$ & $19 \times 1024$ & $\rightarrow$ & $19 \mathrm{x}$ & $19 \times 255$ & 0.189 BFLOPS \\
\hline 24 & & 21 & & $76 \times$ & $76 \times 256$ & $\Rightarrow$ & $76 \times$ & $76 \times 256$ & & 82 & yolo & & & & & - & & & 0.09 diturs \\
\hline 25 & conv & 128 & $1 \times 1 / 1$ & $76 x$ & $76 \times 256$ & $\Rightarrow$ & $76 x$ & $76 \times 128$ & 0.379 BFLOPs & 83 & route & $=79$ & & & & & & & \\
\hline 26 & conv & 256 & $3 \times 3 / 1$ & $76 \times$ & $76 \times 128$ & $\Rightarrow$ & $76 x$ & $76 \times 256$ & 3.407 BFLOPS & 84 & conv & 256 & $1 \times 1 / 1$ & $19 \times$ & $19 \times 512$ & $\rightarrow$ & $19 \times$ & $19 \times 256$ & 0.095 BFLOPS \\
\hline 27 & & 24 & & $76 \times$ & $76 \times 256$ & $\Rightarrow$ & $76 \times$ & $76 \times 256$ & & 85 & upsamp & & & $19 \times$ & $19 \times 256$ & $\rightarrow$ & $38 x$ & $38 \times 256$ & \\
\hline 28 & conv & 128 & $1 \times 1 / 1$ & $76 x$ & $76 \times 256$ & $->$ & $76 x$ & $76 \times 128$ & 0.379 BFLOPs & 86 & route & e 8561 & & & & & & & \\
\hline 29 & conv & 256 & $3 \times 3 / 1$ & $76 \times$ & $76 \times 128$ & $\Rightarrow$ & $76 \times$ & $76 \times 256$ & 3.407 BFLOPs & 87 & conv & 256 & $1 \times 1 / 1$ & $38 \times$ & $38 \times 768$ & $\Rightarrow$ & $38 \times$ & $38 \times 256$ & 0.568 BFLOPS \\
\hline 30 & & 27 & & $76 \times$ & $76 \times 256$ & $\rightarrow$ & $76 x$ & $76 \times 256$ & & 88 & conv & 512 & $3 \times 31$ & $38 \times$ & $38 \times 256$ & $\rightarrow$ & $38 x$ & $38 \times 512$ & 3.407 BFLOPS \\
\hline 31 & conv & 128 & $1 \times 1 / 1$ & $76 \times$ & $76 \times 256$ & $->$ & $76 \times$ & $76 \times 128$ & 0.379 BFLOPs & 89 & conv & 256 & $1 \times 1$ & $38 \times$ & $38 \times 512$ & $\rightarrow$ & $38 x$ & $38 \times 256$ & 0.379 BFLOPS \\
\hline 32 & conv & 256 & $\times 3 / 1$ & $76 x$ & $76 \times 128$ & $\Rightarrow$ & $76 \times$ & $76 \times 256$ & 3.407 BFLOPs & 90 & conv & 512 & $3 \times 3 / 1$ & $38 \times$ & $38 \times 256$ & $\rightarrow$ & $38 \times$ & $38 \times 512$ & 3.407 BFLOPS \\
\hline 33 & res & 30 & & $76 x$ & $76 \times 256$ & $\Rightarrow$ & $76 x$ & $76 \times 256$ & & 91 & conv & 256 & $1 \times 1 / 1$ & $38 \times$ & $38 \times 512$ & $\rightarrow$ & $38 x$ & $38 \times 256$ & 0.379 BFLOPS \\
\hline 34 & conv & 128 & $1 \times 1 / 1$ & $76 \times$ & $76 \times 256$ & $\Rightarrow$ & $76 x$ & $76 \times 128$ & 0.379 BFLOPS & 92 & conv & 512 & $3 \times 3 / 1$ & $38 \times$ & $38 \times 256$ & $\rightarrow$ & $38 \hat{x}$ & $38 \times 512$ & 3.407 BFLOPS \\
\hline 35 & conv & 256 & $\times 3 / 1$ & $76 \times$ & $76 \times 128$ & $\Rightarrow$ & $76 \times$ & $76 \times 256$ & 3.407 BFLOPs & 93 & conv & 255 & $1 \times 1 / 1$ & $38 \times$ & $38 \times 512$ & $\rightarrow$ & $38 \hat{x}$ & $38 \times 255$ & 0.377 BFLOPS \\
\hline 36 & res & 33 & & $76 x$ & $76 \times 256$ & $->$ & $76 x$ & $76 \times 256$ & & 94 & yolo & & & & & & & & \\
\hline 37 & conv & 512 & $3 \times 3 / 2$ & $76 \times$ & $76 \times 256$ & $\Rightarrow$ & $38 \times$ & $38 \times 512$ & 3.407 BFLOPS & 95 & route & 91 & & & & & & & \\
\hline 38 & conv & 256 & $1 \times 1 / 1$ & $38 \times$ & $38 \times 512$ & $->$ & $38 x$ & $38 \times 256$ & 0.379 BFLOPS & 96 & conv & 128 & $1 \times 1 / 1$ & $38 \times$ & $38 \times 256$ & -> & $38 \times$ & $38 \times 128$ & 0.095 BFLOPS \\
\hline 39 & conv & 512 & $3 \times 3 / 1$ & $38 \mathrm{x}$ & $38 \times 256$ & $->$ & $38 \mathrm{x}$ & $38 \times 512$ & 3.407 BFLOPS & 97 & upsamp & & $2 \mathrm{x}$ & $38 \mathrm{x}$ & $38 \times 128$ & $\rightarrow$ & $76 \hat{x}$ & $76 \times 128$ & \\
\hline 40 & res & 37 & & $38 \times$ & $38 \times 512$ & $\Rightarrow$ & $38 \times$ & $38 \times 512$ & & 98 & route & e 9736 & & & & & & & \\
\hline 41 & conv & 256 & $1 \times 1 / 1$ & $38 \times$ & $38 \times 512$ &.$>$ & $38 \times$ & $38 \times 256$ & 0.379 BFLOPs & 99 & conv & 128 & $1 \times 1$ & & $76 \times 384$ & $\Rightarrow$ & $76 \times$ & & 0.568 BFLOPS \\
\hline 42 & conv & 512 & $3 \times 3 / 1$ & $38 \times$ & $38 \times 256$ & $->$ & $38 x$ & $38 \times 512$ & 3.407 BFLOPS & 100 & conv & 256 & & $76 \times$ & $76 \times 128$ & $\Rightarrow$ & $76 x$ & $76 \times 256$ & 3.407 BFLOPS \\
\hline $\begin{array}{l}42 \\
43\end{array}$ & res & $40^{512}$ & & $38 \times$ & 列 $38 \times 512$ & $\vec{~} \rightarrow$ & $38 \times$ & $\begin{array}{l}38 \times 512 \\
38 \times 512\end{array}$ & & 101 & conv & 128 & $1 \times 1 / 1$ & $\begin{array}{l}76 \times x \\
76 \times\end{array}$ & $76 \times 256$ & $\vec{~} \rightarrow$ & $\begin{array}{l}10 \times \\
76 \times\end{array}$ & $76 \times 128$ & O.379 BFLOPS \\
\hline 44 & conv & 256 & $1 \times 1 / 1$ & $38 \times$ & $38 \times 512$ & $\Rightarrow$ & $38 \times$ & $38 \times 256$ & 0.379 BFLOPS & 102 & conv & 256 & $3 \times 3 / 1$ & $76 \times$ & $76 \times 128$ & $\Rightarrow$ & $76 \times$ & $76 \times 256$ & 3.407 BFLOPs \\
\hline 45 & conv & 512 & & $38 \times$ & $38 \times 256$ & $\Rightarrow$ & $38 \times$ & $38 \times 512$ & 3.407 BFLOPS & 103 & conv & 128 & $1 \times 1 / 1$ & $76 \times$ & $76 \times 256$ & $\Rightarrow$ & $76 \times$ & & 0.379 BFLOPS \\
\hline 46 & res & 43 & & $38 \times$ & $38 \times 512$ & $\Rightarrow$ & $38 \mathrm{x}$ & $38 \times 512$ & & 104 & conv & 256 & $3 \times 3 / 1$ & $76 x$ & $76 \times 128$ & 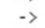 & $76 x$ & $76 \times 256$ & 3.407 BFLOPS \\
\hline 47 & conv & 256 & $1 \times 1 / 1$ & $38 \mathrm{x}$ & $38 \times 512$ & $\rightarrow$ & $38 \mathrm{x}$ & $38 \times 256$ & 0.379 BFLOPS & 105 & conv & 255 & $1 \times 1 / 1$ & $76 \times$ & $76 \times 256$ & $\Rightarrow$ & $76 \times$ & $76 \times 255$ & 0.754 BFLOPS \\
\hline 48 & conv & 512 & $3 \times 3 / 1$ & $38 \times$ & $38 \times 256$ & $\Rightarrow$ & $38 \times$ & $38 \times 512$ & 3.407 BFLOPS & 106 & yolo & & & & & & & & \\
\hline 49 & res & 46 & & $38 \times$ & $38 \times 512$ & $\Rightarrow$ & $38 \mathrm{x}$ & $38 \times 512$ & & & & weight & & & & & & & \\
\hline 50 & conv & 256 & $1 \times 1 / 1$ & $38 \times$ & $38 \times 512$ & $\Rightarrow$ & $38 \times$ & $38 \times 256$ & 0.379 BFLOPS & & & & & & & & & & \\
\hline 51 & conv & 512 & & $38 \times$ & $38 \times 256$ & $\Rightarrow$ & $38 \times$ & $38 \times 512$ & $3.407 \mathrm{BFLC}$ & & & & & & & & & & \\
\hline 52 & res & $49^{12}$ & & $38 \times$ & $38 \times 512$ & $\Rightarrow$ & $38 \mathrm{x}$ & $38 \times 512$ & & & & & $\begin{array}{l}7.1 .7 \% \text { train } \\
1.2 \% \\
\text { testi }\end{array}$ & toss: 28 & & & & & \\
\hline 53 & & 256 & $1 \times 1 /$ & $38 \times$ & $38 \times 512$ & $\rightarrow$ & $38 \mathrm{x}$ & $38 \times 256$ & 0.379 BFLOPS & & ting acc & ccuracy. & & & & & & & \\
\hline 54 & & 512 & & $38 \times$ & $38 \times 256$ & -> & $38 \times$ & $38 \times 512$ & & & & & & & & & & & \\
\hline 55 & & 52 & & 38 & $38 \times 512$ & $\rightarrow$ & 38 & $38 \times 512$ & & & & & & & & & & & \\
\hline 56 & conv & 256 & $1 \times 1 / 1$ & $38 \times$ & $38 \times 512$ & $\rightarrow$ & $38 \times$ & $38 \times 256$ & 0.379 BFLOPS & & & & & T & - & & & & \\
\hline 57 & conv & 512 & $3 \times 3 / 1$ & $38 \times$ & $38 \times 256$ & -> & $38 \times$ & $38 \times 512$ & 3.407 BFLOPS & & & & & & & & & & \\
\hline
\end{tabular}

the Google Firebase and generate various timestamp-based daily, weekly, and monthly reports in both scenarios.

\subsection{The Detailed Working of the Thermal-Scanning Methodology}

The thermal scanning feature of a presented smart kiosk starts functioning when an individual is detected in the projection area of a VGA camera. Once an individual is detected, the presented system will scan their body temperature using a thermal sensor and compare it with the standard body temperature threshold such as $98.6^{\circ} \mathrm{F}\left(37^{\circ} \mathrm{C}\right)$. If the presented kiosk records body temperature of an individual beyond the prescribed limits, it will immediately notify the security control room for further action. Otherwise, timestamp-based records of scanned body temperatures will be stored in Google Firebase for further analysis in both scenarios. The YOLOV4 methodology is divided into two parts: (i) the first part bypasses the dense block and directly moves toward the transition block. (ii) The second part goes through the dense block and reaches the transition block. This kind of design increases the efficiency of the YOLOv4 algorithm by reducing the complexity of the presented approach. In addition to this, the YOLOV4 algorithm also utilizes crossstage-partial connections with CSPDarknet-53 feature extraction methodology, which enables the presented algorithm to achieve very high objection detection accuracy. Figure 7 represents a YOLOv4 methodology, and Figure 8 represents a detailed workflow of the presented smart face-detection and thermal scanning kiosk.

\section{RESULTS AND DISCUSSIONS}

In this section, a detailed discussion of the obtained results of the presented smart face-mask and thermal scanning kiosk for two different approaches (i) the YOLOv4 based face-mask detection methodology (ii) thermopile temperature sensor VGA camera-based body temperature detection methodology. 

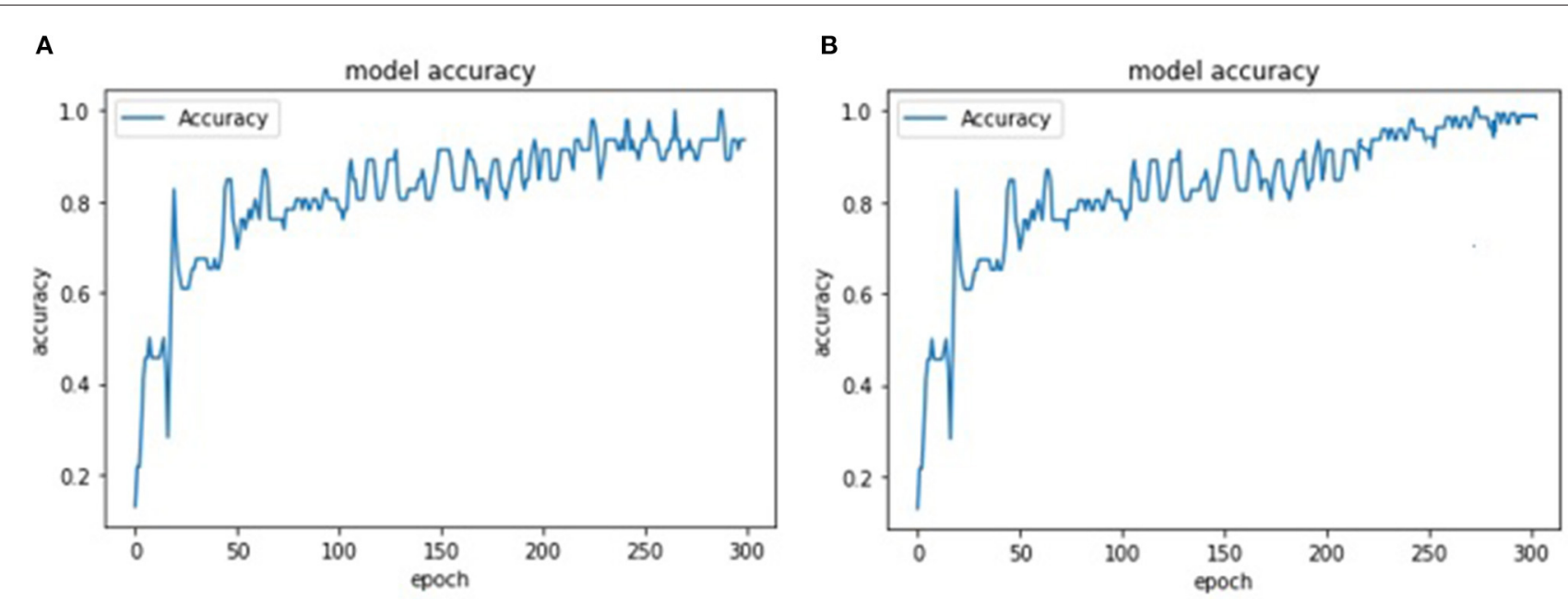

FIGURE 9 | A comparison of a YOLOv4 training and testing accuracy metrics (A) training accuracy (B) testing accuracy.

A

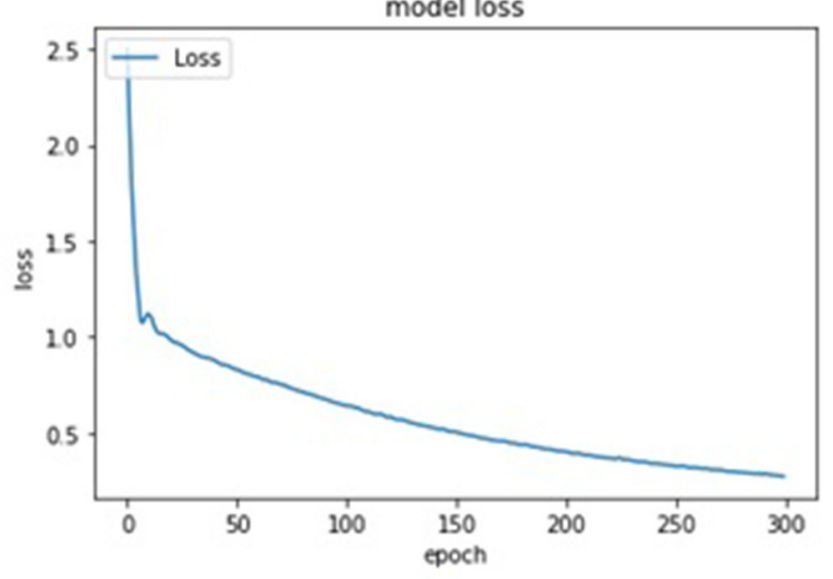

B

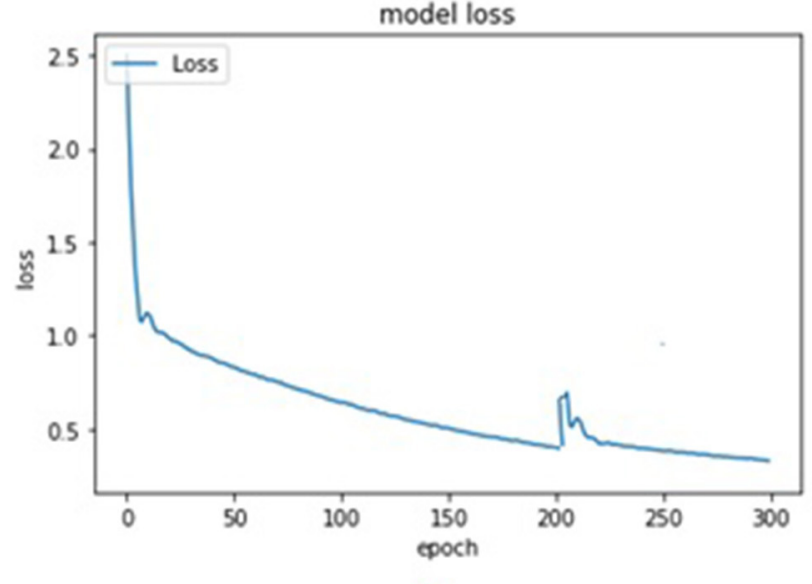

FIGURE 10 | A comparison of a YOLOv4 training and testing accuracy metrics (A) training loss (B) testing loss.

\subsection{Results Discussion of the YOLOv4 Based Face-Mask Detection Methodology}

Figure 9 represents training and testing snapshots of the presented YOLOv4 deep learning methodology. A customized dataset of 1,000 face images was used in the conducted experiments and trained using the YOLOv4 methodology. As described in section 5.2.1, in the conducted experiments, we have used CSPDarknet-53 feature extraction methodology along with the presented YOLOv4 deep learning approach. As shown in Figure 9, we have modified the presented YOLOv4 algorithm to achieve high accuracy in detecting individuals with and without face-mask conditions. Based on the conducted experiments, the presented YOLOV4 methodology has recorded face-mask detection training and testing accuracy of 97.17 and $96.12 \%$, as shown in Figure 10. As shown in Figure 11, based on the conducted experiments, the presented YOLOv4 deep learning algorithm has recorded very minimal training and testing loss, around 1.83 and $2.88 \%$, respectively. Figure 12 depicts face-mask detection samples detected using the YOLOv4 algorithm.

\subsection{Results Discussion of the Thermal Scanning Based Body Temperature Detection Methodology}

In the conducted experiments, we have performed real-time testing of more than 800 individuals to validate the performance of the presented body temperature detection methodology. The presented methodology has utilized a Robocraze OV7670 300KP VGA camera and thermopile temperature sensor to identify individuals exceeding the prescribed standard threshold such as $98.6^{\circ} \mathrm{F}\left(37^{\circ} \mathrm{C}\right)$. Figure 13 represents the real-time thermal scanning results of more than 800 individuals concerning the recorded body temperature. Furthermore, it also depicts the 

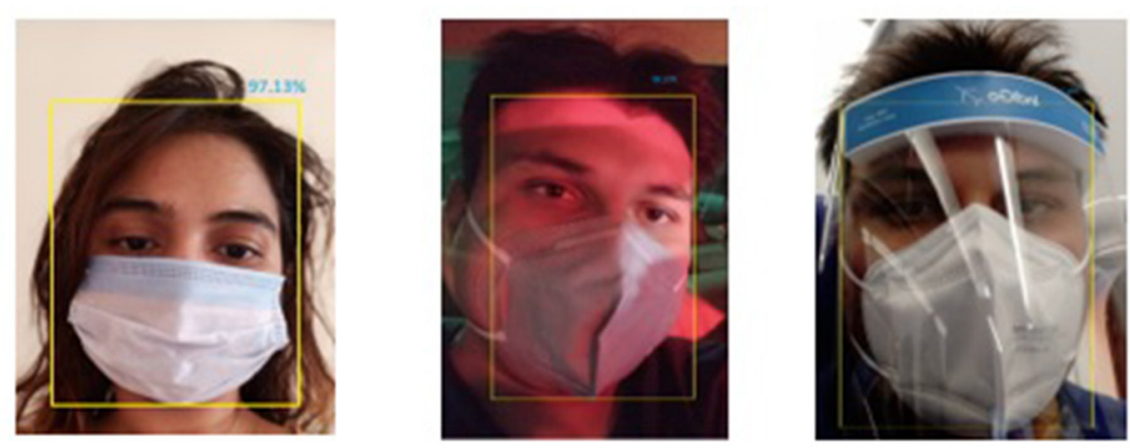

FIGURE 11 | A testing sample of YOLOv4 face-mask detection.
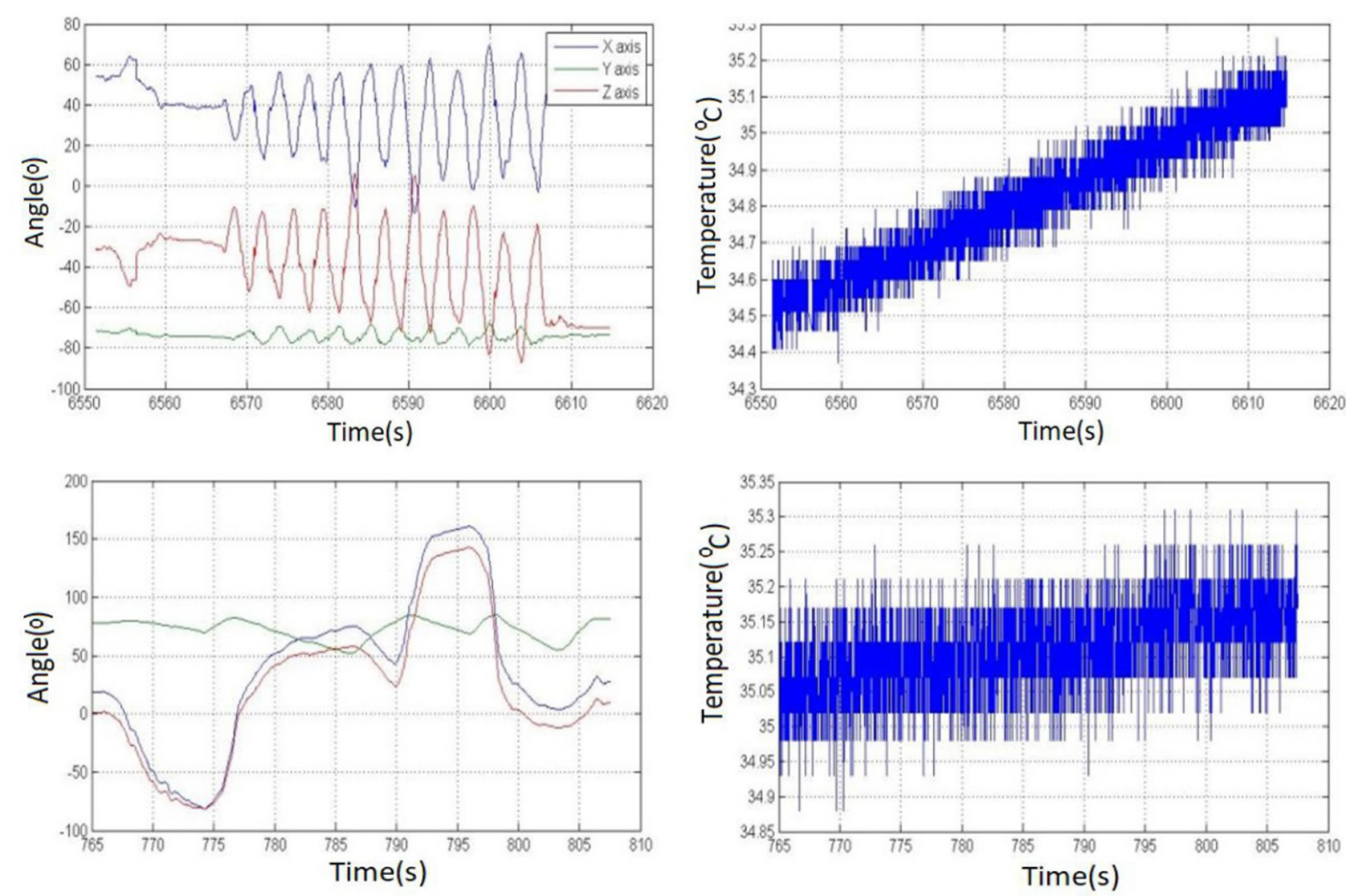

FIGURE 12 | Real-time thermal scanning results of more than 800 individuals.

graphical representation of the number of individuals who a VGA camera has captured from various angles. The presented thermal scanning mechanism has recorded a validation accuracy of $92.01 \%$ thermal scanning kiosk. Furthermore, the presented interfaces present the daily, weekly, and monthly reports of the counts of individuals, along with in-out timestamps fetched from the google firebase cloud computing platform. Furthermore, it also keeps track of the consumed power and also provides information on power usage reports.

\subsection{The Web and Mobile Interface of a Smart Face-Mask and Thermal Scanning Kiosk}

Figure 13 represents a GUI-based web and mobile interface design of the presented smart face-mask and thermal scanning kiosk. The presented interfaces present the daily, weekly, and monthly reports of the counts of individuals, along with in-out timestamps fetched from the google firebase cloud computing 


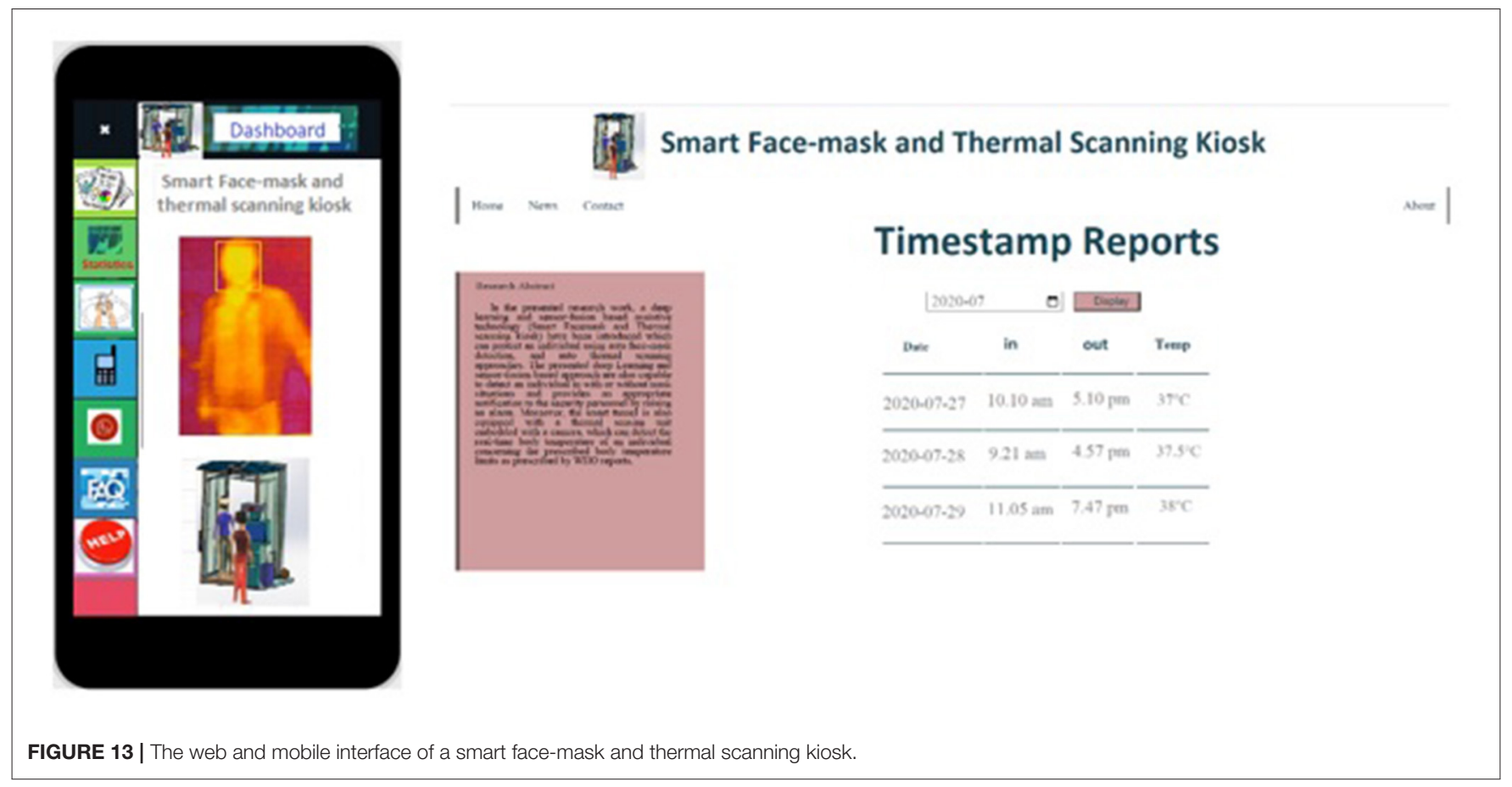

platform. Furthermore, it also keeps track of the consumed power and also provides information on power usage reports.

\section{CONCLUSION AND FUTURE ENHANCEMENTS}

This study presents a deep learning and sensor-fusion-based assistive technology (Smart Facemask and Thermal scanning kiosk) to detect individuals with and without mask conditions. The presented research work is an extended version of the smart epidemic tunnel presented previously. In the undertaken study, the presented smart face-mask and thermal scanning kiosk have facilitated two essential tasks (i) the bifurcation of the people based on a variety of face mask conditions such as with and without face mask conditions using a YOLOv4 computer vision algorithm deployed in an NVIDIA Netson controller. In addition, (ii) it can also detect the body temperature of individuals present in the projection areas of a Robocraze OV7670 300KP VGA camera using a Thermopylae temperature sensing unit. The investigation results validate the performance evaluation of the presented smart face-mask and thermal scanning mechanism. The major findings of this study are as follows: (i) The presented system can detect an outsider who is entering the building with or without mask conditions and notifies the security control room by raising appropriate alarms. (ii) The presented smart face-mask detection and thermal scanning kiosk can function using solar energy during the day, and it functions using a solar power bank at night. This functionality has been provided by an LDR sensing unit placed in a tunnel. (iii) The presented smart kiosk also provides the facility to do real-time timestamp-based attendance of all the staff entering the smart tunnel, along with thermal scanning of the body temperature and face-mask detection. Based on the experiments, the presented YOLOV4 methodology recorded face-mask detection training and testing accuracy of 97.17 and $96.12 \%$. Furthermore, the presented thermal scanning mechanism has recorded a validation accuracy of $92.01 \%$. (iv) In the end, web and mobile interface has been designed to provide daily, weekly, and monthly reports of the recorded body temperatures of individuals, along with in-out timestamp values. In the future, the proposed research work can be extended with various scenarios, such as obscured faces, half, or fully obscured human faces with plastic, leather, or any other materials.

\section{DATA AVAILABILITY STATEMENT}

The raw data supporting the conclusions of this article will be made available by the authors, without undue reservation.

\section{ETHICS STATEMENT}

Written informed consent was obtained from the individual(s), and minor(s)' legal guardian/next of kin, for the publication of any potentially identifiable images or data included in this article.

\section{AUTHOR CONTRIBUTIONS}

SP and AS: conceptualization and data collection and interpretation and data curation. SP, AS, and NS: manuscript editing. All authors contributed to the article and approved the submitted version. 


\section{REFERENCES}

1. Ayoub A, Mahboob K, Javed AR, Rizwan M, Gadekallu TR, Abidi MH, et al. Classification and categorization of COVID-19 outbreak in Pakistan. Comput Mater Continua. (2021) 69:1253-69. doi: 10.32604/cmc.2021.015655

2. Ghayvat H, Awais M, Gope P, Pandya S, Majumdar S. Recognizing suspect and predicting the spread of contagion based on mobile phone location data (counteract): a system of identifying covid-19 infectious and hazardous sites, detecting disease outbreaks based on the internet of things, edge computing, and artificial intelligence. Sus Cities Soc. (2021) 69:102798. doi: $10.1016 /$ j.scs.2021.102798

3. Pandya S, Ghayvat H. Ambient acoustic event assistive framework for identification, detection, and recognition of unknown acoustic events of a residence. Adv Eng Inform. (2021) 47:101238. doi: 10.1016/j.aei.2020.101238

4. Aslam B, Javed AR, Chakraborty C, Nebhen J, Raqib S, Rizwan M. Blockchain and ANFIS empowered IoMT application for privacy preserved contact tracing in COVID-19 pandemic. Pers Ubiquitous Comput. (2021) 22:1-17. doi: 10.1007/s00779-021-01596-3

5. Bhattacharya S, Maddikunta PKR, Pham QV, Gadekallu TR, Chowdhary CL, Alazab M, et al. Deep learning and medical image processing for coronavirus (COVID-19) pandemic: a survey. Sus Cities Soc. (2021) 65:102589. doi: $10.1016 /$ j.scs.2020.102589

6. Garg D, Goel P, Pandya S, Ganatra A, Kotecha K. A deep learning approach for face detection using YOLO. In 2018 IEEE Punecon. Pune: IEEE (2018). p. $1-4$.

7. Ghayvat H, Pandya SN, Bhattacharya P, Zuhair M, Rashid M, Hakak S, et al. CP-BDHCA: blockchain-based confidentiality-privacy preserving big data scheme for healthcare clouds and applications. IEEE J Biomed Health Inform. (2021) doi: 10.1109/JBHI.2021.3097237

8. Pandya S, Sur A, Kotecha K. Smart epidemic tunnel: IoT-based sensorfusion assistive technology for COVID-19 disinfection. Int J Pervasive Comput Commun. (2020) doi: 10.1108/IJPCC-07-2020-0091

9. Sur A, Sah RP, Pandya S. Milk storage system for remote areas using solar thermal energy and adsorption cooling. Mater Today Proc. (2020) 28:1764-70. doi: 10.1016/j.matpr.2020.05.170

10. Srivastava A, Jain S, Miranda R, Patil S, Pandya S, Kotecha K. Deep learning based respiratory sound analysis for detection of chronic obstructive pulmonary disease. PeerJ Comput Sci. (2021) 7:e369. doi: 10.7717/peerj-cs.369

11. Ghayvat H, Pandya S, Shah S, Mukhopadhyay S, Yap M, Wandra K. Advanced AODV approach for efficient detection and mitigation of wormhole attack in MANET. In: 2016 10th International Conference on Sensing Technology (ICST). Nanjing: IEEE (2016). p. 1-6.

12. Yang L, Liu J, Tang X. Object detection and viewpoint estimation with auto-masking neural network. In: European Conference on Computer Vision. Glasgow: Springer (2014). p. 441-55.

13. Joshi JR. COVSACK: an innovative portable isolated and safe COVID-19 sample collection kiosk with automatic disinfection. Trans Indian Natl Acad Eng. (2020) 5:269-75. doi: 10.1007/s41403-020-00139-1

14. Fan DP, Zhou T, Ji GP, Zhou Y, Chen G, Fu H, et al. Inf-net: Automatic covid19 lung infection segmentation from ct images. IEEE Trans Med Imaging. (2020) 39:2626-37. doi: 10.1109/TMI.2020.2996645

15. Maurya D, Gohil MK, Sonawane U, Kumar D, Awasthi A, Prajapati AK, et al. Development of autonomous advanced disinfection tunnel to tackle external surface disinfection of COVID-19 virus in public places. Trans Indian Natl Acad Eng. (2020) 5:281-7. doi: 10.1007/s41403-020-00141-7

16. Abbas A, Abdelsamea MM, Gaber MM. Classification of COVID-19 in chest X-ray images using DeTraC deep convolutional neural network. Appl Intell. (2021) 51:854-64. doi: 10.1007/s10489-020-01829-7

17. Apostolopoulos ID, Mpesiana TA. Covid-19: automatic detection from $\mathrm{x}$-ray images utilizing transfer learning with convolutional neural networks. Phys Eng Sci Med. (2020) 43:635-40. doi: 10.1007/s13246-02000865-4

18. Poon L, Abramowicz J, Dall'Asta A, Sande R, Ter Haar G, Maršal K, et al. ISUOG safety committee position statement on safe performance of obstetric and gynecological scans and equipment cleaning in context of COVID-19. Ultrasound ObstetrGynecol. (2020) 55:709-12. doi: 10.1002/uog.22027

19. Kim SI, Lee JY. Walk-through screening center for COVID-19: an accessible and efficient screening system in a pandemic situation. J Korean Med Sci. (2020) 35:e154. doi: 10.3346/jkms.2020.35.e154

20. Ucar F, Korkmaz D. COVIDiagnosis-Net: deep bayes-squeezenet based diagnosis of the coronavirus disease 2019 (COVID-19) from X-ray images. Med Hypotheses. (2020) 140:109761. doi: 10.1016/j.mehy.2020.109761

21. Kwon KT, Ko JH, Shin H, Sung M, Kim JY. Drive-through screening center for COVID-19: a safe and efficient screening system against massive community outbreak. J Korean Med Sci. (2020) 35:e123. doi: 10.3346/jkms.2020.35.e123

22. El Majid B, Motahhir S, El Hammoumi A, Lebbadi A, El Ghzizal A. Preliminary design of a smart wristband disinfectant to help in covid-19 fight. Inventions. (2020) 5:32. doi: 10.3390/inventions5030032

23. Takagi G, Yagishita K. Principles of disinfectant use and safety operation in medical facilities during coronavirus disease 2019. (COVID-19) outbreak. SN Comprehens Clin Med. (2020) 2:1041-4. doi: 10.1007/s42399-020-00413-x

24. Ullah F, Haq HU, Khan J, Safeer AA, Asif U, Lee S. Wearable IoTs and geofencing based framework for COVID-19 remote patient health monitoring and quarantine management to control the pandemic. Electronics. (2021) 10:2035. doi: 10.3390/electronics 10162035

25. Lippi G, Adeli K, Ferrari M, Horvath AR, Koch D, Sethi S, et al. Biosafety measures for preventing infection from COVID-19 in clinical laboratories: IFCC taskforce recommendations. Clin Chem Lab Med. (2020) 58:1053-62. doi: $10.1515 / \mathrm{cclm}-2020-0633$

26. Mahammedi NA, Mahammedi A. Standalone solar-powered ultraviolet mobile disinfectant: bringing solar energy in the global fight against COVID-19. Preprints. (2020) 2020:2020050415. doi: 10.20944/preprints202005.0415.v1

27. Pandya S, Ghayvat H, Kotecha K, Awais M, Akbarzadeh S, Gope P, et al. Smart home anti-theft system: a novel approach for near real-time monitoring and smart home security for wellness protocol. Appl Syst Innov. (2018) 1:42. doi: $10.3390 /$ asi1040042

28. Ghayvat H, Awais M, Pandya S, Ren H, Akbarzadeh S, Chandra Mukhopadhyay S, et al. Smart aging system: uncovering the hidden wellness parameter for well-being monitoring and anomaly detection. Sensors. (2019) 19:766. doi: 10.3390/s19040766

29. Sengupta S. YOLO Application in COVID-19 Use Cases. (2021).

Conflict of Interest: The authors declare that the research was conducted in the absence of any commercial or financial relationships that could be construed as a potential conflict of interest.

Publisher's Note: All claims expressed in this article are solely those of the authors and do not necessarily represent those of their affiliated organizations, or those of the publisher, the editors and the reviewers. Any product that may be evaluated in this article, or claim that may be made by its manufacturer, is not guaranteed or endorsed by the publisher.

Copyright $\odot 2021$ Pandya, Sur and Solke. This is an open-access article distributed under the terms of the Creative Commons Attribution License (CC BY). The use, distribution or reproduction in other forums is permitted, provided the original author(s) and the copyright owner(s) are credited and that the original publication in this journal is cited, in accordance with accepted academic practice. No use, distribution or reproduction is permitted which does not comply with these terms. 